\title{
Comparison of Laparoscopic Myomectomy with and without Uterine Artery Occlusion in Treatment of Symptomatic Multiple Myomas
}

\section{YanZhen Peng \\ JiuMei Cheng \\ ChunYi Zang \\ Xi Chen \\ JinXue Wang}

Department of Minimally Invasive Gynecology, Beijing Obstetrics and Gynecology Hospital, Capital Medical University, Beijing, 100006, People's Republic of China
Correspondence: JiuMei Cheng Department of Minimally Invasive Gynecology, Beijing Obstetrics and Gynecology Hospital Capital Medical University, No. 17 Qihelou Street, Dongcheng District, Beijing, 100006, People's Republic of China Tel +86 I8600245876

Email cjmI429@ccmu.edu.cn
Objective: Uterine artery occlusion (UAO) is a minimally invasive approach often used to treat symptomatic uterine myomas. This study aimed to compare the clinical effects of laparoscopic UAO (LUAO) in combination with laparoscopic myomectomy (LM) with LM alone to treat symptomatic multiple uterine myomas.

Methods: This was a prospective observational study. In total, 122 patients with symptomatic multiple uterine myomas underwent LUAO + LM or LM alone between April 2015 and October 2017. The surgical procedure time, blood loss, highest postoperative temperature, hospital length of stay, number of removed myomas, surgical complications, and recurrence rate of the two groups were compared.

Results: Mean blood loss was significantly lower in the LUAO + LM group compared with the LM group $(177.97 \pm 104.09 \mathrm{~mL}$ vs $258.10 \pm 119.55 \mathrm{~mL}, \mathrm{p}<0.05)$. No significant difference in surgical procedure time, hospital length of stay, highest postoperative temperature, and surgical complications was found between the LUAO + LM group and LM group. The number of removed myomas was considerably higher in the LUAO + LM group than in the LM group (4[4-7] vs 3[3-5], p < 0.05). The recurrence rate in the LUAO + LM group was considerably lower than that in the LM group $(6.2 \%$ vs $25.9 \%$ ).

Conclusion: LUAO in combination with LM was associated with higher surgical quality and lower recurrence of myomas compared with LM alone. LUAO in combination with LM is recommended for women with symptomatic multiple uterine myomas who wish to retain their uteruses.

Keywords: laparoscopic myomectomy, uterine artery occlusion, uterine myomas

\section{Introduction}

Uterine myoma is the most common female reproductive system benign tumor, with an incidence of $20-40 \%$ in women of reproductive age. ${ }^{1}$ Patients with symptomatic myomas usually suffer from menorrhagia, pelvic pain, urinary tract and/or bowel pressure symptoms, and infertility. The surgical approach remains the mainstay of treatment for symptomatic myomas. ${ }^{2}$ Although hysterectomy, a conventional treatment, provided definite therapeutic effectiveness for women who did not wish to preserve their fertility, there is currently a trend toward retaining the uterus and adopting a minimally invasive approach in the surgical treatment of uterine myomas. ${ }^{1}$ Furthermore, with the improvement in surgeons' skills and the development of laparoscopic instruments, laparoscopic myomectomy (LM) has been widely 
adopted for patients who wish to preserve their uterus regardless of their desire to preserve fertility.

Although LM has more advantages, such as shorter hospital length of stay (LOS), quicker recovery after surgery, and a better cosmetic result than laparotomy, the morbidity related to the surgery cannot be neglected. Factors such as intraoperative excessive blood loss, prolonged operating time, and increased likelihood of myoma recurrence were frequently encountered. Therefore, in recent years, the combination of LM and permanent or transient laparoscopic uterine artery occlusion (LUAO) has been advocated. A growing body of evidence has confirmed that the positive clinical outcomes after LM + LUAO are reduced intraoperative blood loss and recurrence risk. ${ }^{3,4}$ However, the majority of previous studies focused only on uncomplicated LM based on the conception that when faced with myomas with a diameter larger than $8 \mathrm{~cm}$ or with multiple leiomyomas $(>3)$, a myomectomy by laparotomy may be more appropriate. ${ }^{5,6} \mathrm{~A}$ more recent study found that retaining the uterus and adopting the minimally invasive approach were the most important changes in the development of surgical treatment of uterine myomas. Endoscopic surgery has gradually replaced traditional laparotomy. ${ }^{1}$

This observational study aimed to evaluate the safety and efficacy of LM + LUAO in the treatment of women with symptomatic multiple uterine myomas compared with LM alone.

\section{Patients and Methods}

\section{Patients}

This was a prospective observational study of patients with symptomatic multiple myomas that necessitated conservative surgery, which was conducted in the Center of Minimally Invasive Gynecological Surgery of Beijing Obstetrics and Gynecology Hospital between April 2015 and October 2017. The uterine myomas were diagnosed by transvaginal ultrasound. Myoma characteristics, such as the type, number, site (anterior, posterior, fundal), and diameter of dominant myomas, and three diameters of uterine were recorded. Uterine volumes were calculated by the formula: (upper and lower diameter $\times$ front and rear diameter $\times$ left and right diameter $\times 0.523$. The symptoms attributed to myomas included menorrhagia, frequency of urination, and a bulge-like sensation. Inclusion criteria for the study were as follows: two or more symptomatic intramural myomas (FIGO type 3,4,5,6) regardless of simultaneous subserosal fibroids (FIGO type 7), ${ }^{7}$ absence of previous abdominal or pelvic surgery, and a strong desire to retain the uterus. Patients were excluded if they had submucosal myomas or malignant conditions confirmed by postoperative pathological results.

Finally, 112 patients were consecutively enrolled, and their general characteristics (age and body mass index [BMI]) and maternal history were recorded. Preoperatively, all patients underwent a diagnostic examination to exclude other diseases. Patients who did not wish to preserve their fertility underwent LM combined with UAO (LM + LUAO group, $\mathrm{n}=64)$, whereas those wishing to preserve their fertility were treated with LM alone (LM group, $\mathrm{n}=58$ ). All operations were performed by one surgeon. Written informed consent was obtained from each patient, and all of the patients were followed regularly for two years postoperatively.

\section{Operative Procedures}

All patients were placed in a dorsolithotomy position with the bladder catheterized. General anesthesia was induced with endotracheal intubation. A uterine manipulator was used to allow uterine movement. After a laparoscopic surgical field was established, the intra-abdominal pressure was maintained between 12 and $14 \mathrm{mmHg}$ with $\mathrm{CO}_{2}$.

For the patients in the LUAO + LM group, the first step of the surgical procedure was bilateral occlusion of the uterine artery. In most cases, an incision of approximately $3 \mathrm{~cm}$ was made on the posterior leaf of the broad ligament above the uterosacral ligaments approximately $2 \mathrm{~cm}$ from the uterine isthmus. Two other approaches can be adopted for peritoneal incisions. In the first approach, the incision is made in the triangle area in the broad ligament (enclosed by the round ligament, the infundibulopelvic ligament, and the external iliac vessels), and in the second approach, the incision is made in the anterior leaf of the broad ligament (Figure 1). The choice of incision approach depends on the volume of the uterus, position and size of dominant myomas, pelvic space, and formation of pelvic adhesions. When enlarged volume of the uterus, dominant myomas located on posterior wall, or formation of pelvic adhesions induced difficult expose of posterior leaf in the broad ligament, the triangle area or anterior leaf approach was adopted. Whichever approach was chosen, the ureter and the uterine artery were identified and carefully separated by blunt dissection. Subsequently, the uterine artery was thoroughly occluded by being coagulated with bipolar forceps with power 


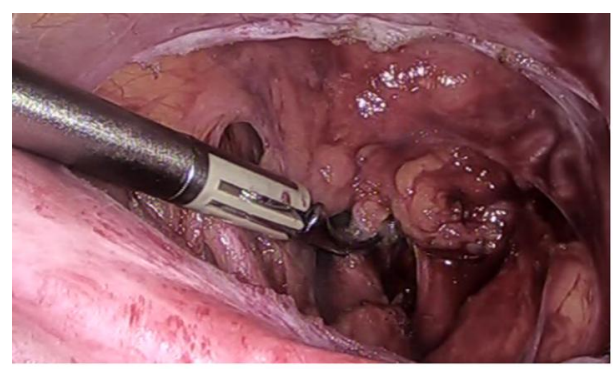

A

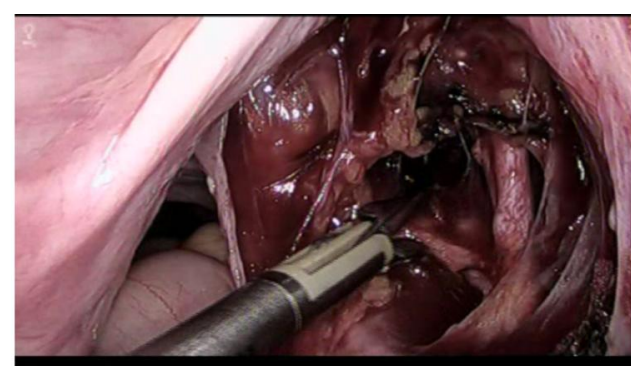

B

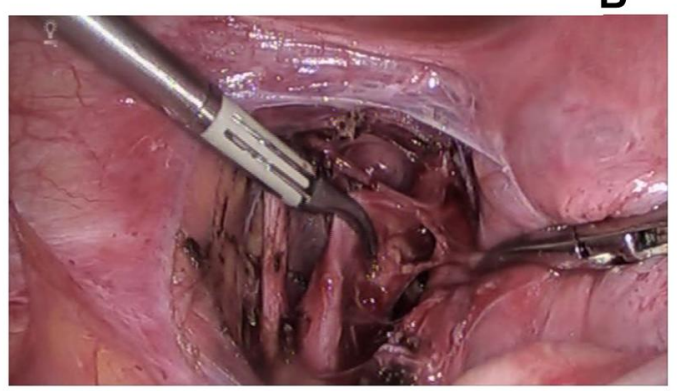

C

Figure I Three different approaches of laparoscopic uterine artery occlusion(LUAO). (A) The anterior leaf of the broad ligament. (B) The posterior leaf of the broad ligament. (C) The triangle area in the broad ligament.

between 40 and $45 \mathrm{~W}$ under direct laparoscopic vision. The coagulation bandwidth was $1.0-1.5 \mathrm{~cm}$. The characteristics of the uterine artery included the location 1-2 cm above the ureter, diameter of 2-6 $\mathrm{mm}$, tortuous shape, and vascular pulsation.

The LM for the patients in both groups was carried out as follows. First, dilute vasopressin $(3 \mathrm{U} / 100 \mathrm{~mL})$ was injected into the myometrium with an injector under intense vital sign monitoring. Then, an incision through the superficial myometrium and the pseudocapsule was made with a monopolar electric hook until the myoma was visually exposed. A screw forceps was used to fix and distract the leiomyoma, which was then separated from the uterus. The myometrial defect was repaired using 1-0 continuous absorbable sutures. The excised leiomyoma was removed by an electromechanical morcellator through the $15-\mathrm{mm}$ trocar site located in the right lower abdomen.

\section{Evaluation Parameters}

Intraoperative parameters we considered for comparing the two groups included surgical procedure time, estimated blood loss, conversion to laparotomy, number of removed myomas, and blood transfusion. The surgical procedure time was calculated from the first abdomen wall incision to the closure of all laparoscopic incisions, which was recorded by the anesthetist. Blood loss was estimated based on the volume of fluids collected in the suction minus the volume of rinsing. Several postoperative outcomes were evaluated, such as hospital LOS, hemoglobin drop $(\mathrm{HgD})$ at postoperative day two, and highest postoperative temperature. Perioperative complications included wound infection, deep vein thrombosis, intestinal injuries or obstruction, and urinary system injuries.

Patients were followed up for at least two years and asked to return to the hospital every 3-6 months after surgery. The relieving of myoma-related symptoms was recorded, and transvaginal ultrasound was undertaken. Symptomatic myomas of $\geq 2 \mathrm{~cm}$ confirmed by ultrasound examination were regarded as myoma recurrence. The recurrence rate was determined at two years following the operation.

\section{Statistical Analysis}

SPSS Version 19.0 for Windows (SPSS Inc., Chicago, IL, USA) was used for the statistical analysis. All continuous variables were tested for normality with the KolmogorovSmirnov test. Normally distributed variables were expressed as mean \pm standard deviation, while skewed variables were reported as median and interquartile range. The parametric independent samples $t$-test or the nonparametric Mann-Whitney $U$-test was used to compare 
the difference between the two groups. Categorical variables are presented as percentages and were compared using the Chi-square test or Fisher exact test, as appropriate. All calculated p-values were two-tailed. The level of statistical significance was set at a value of $\mathrm{p}<0.05$.

\section{Results}

In total, 122 patients fulfilling the inclusion criteria with complete surgical and follow-up data were enrolled in the study. Of these, 64 underwent LUAO + LM, and 58 underwent LM alone. The demographic and clinical characteristics of patients and myoma parameters are shown in Table 1. There were no significant differences in gravidity, parity, BMI, main symptoms resulting from myomas, dominant myoma diameter and location, and preoperative hemoglobin level between the two groups.

No case required conversion to laparotomy due to technical difficulty or intraoperative complication. The surgical procedure time was not significantly different between the LUAO + LM group and the LM group. However, the amount of blood loss during the surgery in the LUAO + LM group was significantly less than that in the LM group $(177.97 \pm 104.09 \mathrm{~mL}$ vs $258.10 \pm$ $119.55 \mathrm{~mL}$ ). The number of removed myomas in the LUAO + LM group was significantly higher compared

Table I Baseline Demographic and Clinical Characteristics of Patients

\begin{tabular}{|c|c|c|c|}
\hline Characteristics & $\begin{array}{l}\text { LUAO+LM } \\
(n=64)\end{array}$ & $\operatorname{LM}(n=58)$ & $P$ value \\
\hline Age(years) & $41.20 \pm 3.93$ & $38.89 \pm 4.40$ & 0.030 \\
\hline $\mathrm{BMI}(\mathrm{kg} / \mathrm{m} 2)$ & $23.73(21.09-28.58)$ & $24.21(22.86-28.32)$ & 0.474 \\
\hline Gravidity & $3(1-3)$ & $2(I-4)$ & 0.510 \\
\hline Parity & I (I-2) & $I(I-I)$ & 0.094 \\
\hline Preoperative Hg (g/dL) & $\mid 1.52 \pm 1.71$ & $11.91 \pm 1.76$ & 0.233 \\
\hline \multicolumn{4}{|l|}{ Main Symptom(n.\%) } \\
\hline Menorrhagia & $32(50.0)$ & $24(4 I .4)$ & 0.340 \\
\hline Frequency & $20(31.2)$ & $25(43.1)$ & 0.175 \\
\hline Bulge sensation & $12(18.8)$ & $9(15.5)$ & 0.637 \\
\hline Uterine volume $\left(\mathrm{cm}^{3}\right)$ & $236.67 \pm 116.97$ & $|97.84 \pm 90.8|$ & 0.043 \\
\hline Max.diameter(cm) & $5.72 \pm 1.49$ & $5.42 \pm 1.55$ & 0.314 \\
\hline \multicolumn{4}{|l|}{$\begin{array}{l}\text { Dominant tumor } \\
\text { location(n.\%) }\end{array}$} \\
\hline Anterior & $23(35.9)$ & $30(51.7)$ & 0.079 \\
\hline Posterior & $33(51.6)$ & $22(37.9)$ & 0.131 \\
\hline Fundal & $8(12.5)$ & $6(10.3)$ & 0.709 \\
\hline
\end{tabular}

with the LM group. During or immediately after surgery, two of the patients in the LM group required a blood transfusion, whereas none of the patients in the LUAO + LM group required one.

No major acute or delayed perioperative complications emerged in either group of patients. The $\mathrm{HgD}$ at postoperative day two was statistically significantly lower in the LM group than in the LUAO + LM group (1.33 \pm $0.98 \mathrm{mg} / \mathrm{dL}$ vs $1.75 \pm 1.16 \mathrm{mg} / \mathrm{dL}$ ). No differences were found in the average length of the postoperative hospital stay, the median of which was six days (range: 2-7) for the LUAO + LM group and six days (range: 4-9) for the LM group. There was a statistically significant difference between the two groups in the recurrence rate of myomas. Four patients $(6.2 \%)$ in the LUAO + LM group and 15 patients $(25.9 \%)$ in the LM group had recurrent myomas (see Table 2).

\section{Discussion}

Although non-surgical management strategies for symptomatic uterine myomas, such as uterine artery embolization (UAE), high-frequency magnetic resonance-guided focused ultrasound surgery, and medical therapy, have been established in recent years, ${ }^{2,6,8} \mathrm{LM}$ still is the main minimally invasive treatment of choice for patients who wish to preserve their fertility or uterine organ. However,

Table 2 Surgical and Postoperative Outcomes

\begin{tabular}{|l|l|l|l|}
\hline Variables & $\begin{array}{l}\text { LUAO+LM } \\
(\mathbf{n = 6 4 )}\end{array}$ & LM (n=58) & P value \\
\hline Surgical time (min) & $108.89 \pm 28.80$ & $107.84 \pm 37.63$ & 0.864 \\
\hline Blood loss (mL) & $177.97 \pm 104.09$ & $258.10 \pm 119.55$ & 0.000 \\
\hline $\begin{array}{l}\text { Number of removed } \\
\text { myomas(n.) }\end{array}$ & $4(4-7)$ & $3(3-5)$ & 0.000 \\
\hline $\begin{array}{l}\text { Conversion to } \\
\text { laparotomy(n.) }\end{array}$ & 0 & 0 & - \\
\hline $\begin{array}{l}\text { Blood transfusion(n.) } \\
\text { Postoperative } \\
\text { haemoglobin drop(mg/dL) }\end{array}$ & 0 & 2 & 0.224 \\
\hline $\begin{array}{l}\text { Recurrence rate (n.\%) } \\
\text { complications(n.) }\end{array}$ & $4(6.2)$ & $6(5-6)$ & 0.717 \\
\hline $\begin{array}{l}\text { Highest postoperative } \\
\left.\text { temperature ( }{ }^{\circ} \mathrm{C}\right)\end{array}$ & $37.73 \pm 0.48$ & $37.78 \pm 0.47$ & 0.553 \\
\hline
\end{tabular}


LM is associated with an increased risk of morbidity due to intraoperative excessive bleeding and post-surgery recurrence of symptomatic uterine myomas due to technical difficulty, especially in cases of multiple uterine myomas. ${ }^{6,9} \mathrm{UAO}$, a hemostatic technique, was introduced in 1999. Although some evidence has shown advantages of performing LUAO at the time of myomectomy, such as a reduction in blood loss and a lower recurrence rate, ${ }^{10-12}$ there are conflicting results. ${ }^{4}$ Moreover, few studies have focused on its effect on patients with multiple uterine myomas. Our study aimed to explore the effect of combined LUAO and LM on the management of multiple uterine myomas.

Although several previous studies showed conflicting results regarding the efficacy of LUAO in reducing intraoperative blood loss in LM, a more recent directcomparison meta-analysis of short- and long-term outcomes in women with symptomatic leiomyomas of LUAO combined with LM and LM alone has demonstrated a significant difference in intraoperative blood loss, suggesting that LUAO may have a positive impact on minimizing blood loss. ${ }^{3}$ Our study assessed the hemostatic effect of LUAO on LM in patients with multiple uterine myomas. Results showed significantly less intraoperative blood loss in the LUAO + LM group than in the LM group. Correspondingly, $\mathrm{HgD}$ at postoperative day two was significantly lower in the LUAO + LM group than in the LM group. Therefore, LUAO would be an effective hemostatic technique, especially in patients with multiple myomas who received more than one uterine incision during the operation.

The hemostatic effect of LUAO could provide many benefits in facilitating the surgical procedure and reducing morbidity associated with surgery. First, the absence of excessive bleeding contributed to a clearer surgical field, making the removal of uterine fibroids more feasible and thorough. This was confirmed by our study, which revealed that the number of removed myomas in the LUAO + LM group was higher than that in the LM group. Second, the potential electronic damage to the myometrium was minimized due to the reduced need for bipolar coagulation hemostasis, which could be conducive to more rapid postoperative recovery, as reported by Yang. ${ }^{11}$ Third, less intraoperative blood loss contributed to lower blood transfusion rates and related risks. Therefore, as an effective hemostatic technique, LUAO can be applied in other gynecological laparoscopic surgery, such as adenomyomectomy, ectopic pregnancies in the cesarean scar, and complicated hysterectomy, which may lead to excessive bleeding. ${ }^{13,14}$

The high recurrence rate after LM remains a clinically challenging problem to address, particularly that of multiple uterine myomas, which carry an increased risk of recurrence. ${ }^{15}$ Several previous studies found that LUAO + LM may be more effective than LM alone to reduce the likelihood of recurrence. ${ }^{11,16,17}$ Our study's findings are consistent with this since the LUAO + LM group had a significantly lower recurrence rate than the LM group $(6.2 \%$ vs $25.9 \%, p=0.003)$ at the two-year follow-up. It is currently known there are two reasons for postoperative recurrence of myomas. One is the growth of residual small fibroids that were undetectable during the operation. LUAO results in ischemia and hypoxia, which cause residual fibroids to become necrotic and unable to grow. Several research studies have revealed differences in the coagulation-fibrinolysis system and compensatory blood supply between myomas and the uterus myometrium, which lead to the duration of hypoxia in myomas but restoration of blood supply in the uterus. ${ }^{10,11}$ This may be the therapeutic mechanism of LUAO on myomas, which would account for the lower recurrence after LM. In addition, as shown in our study, due to the homeostatic effect of LUAO, a clearer surgical field facilitated a more thorough removal of myomas, reducing the likelihood of recurrence. Notably, however, the transient LUAO technique was not beneficial in reducing the recurrence of myomas, as reported by Jin. ${ }^{18}$ This implied that only persistent hypoxia-ischemia could cause necrosis of myomas.

Given that LUAO has a similar effect on ischemia of the uterus to UAE, which, as has been proven, may cause impaired ovarian function and insufficient endometrium, concerns regarding the negative impacts of LUAO on ovary reserve and fertility outcome have always existed. Although there is evidence supporting no impaired ovarian function after temporary occlusion, including normal postoperative Doppler values of uterine arteries and antimullerian hormone and follicle-stimulating hormone levels, ${ }^{17,18}$ few studies have focused on permanent occlusion. Some scholars suggest that whether it impacts ovarian function appears to depend on the site of uterine vessel blockage. As reported by Lee, ${ }^{19}$ UAO combined with simultaneous blockage of anastomosis between the uterine and ovarian vessels was associated with a greater risk of a significant increase in follicle-stimulating hormone level in the first month after the operation than UAO alone, which may reflect diminished ovarian function. 
The true fertility consequences of undergoing LUAO remain inconclusive due to the lack of prospective randomized controlled studies, although a few comparative studies have revealed no marked differences in the percentage of pregnancies and children born alive in the occlusion + myomectomy group compared with myomectomy only., ${ }^{4,20,21}$ However, Michal et al, who conducted a prospective nonrandomized clinical trial comparing clinical outcomes of women with fibroid(s) treated with UAE and LUAO, ${ }^{22}$ found that the mean birth weight of neonates was lower ( $3270 \mathrm{~g}$ vs $2768 \mathrm{~g}, \mathrm{p}=0.013$ ), and the incidence of intrauterine growth restriction was higher ( $13 \%$ vs $38 \%, p=0.046)$ in patients who underwent LUAO despite similar results regarding pregnancy $(69 \%$ after UAE vs $67 \%$ after LUAO), delivery ( $50 \%$ vs $46 \%$ ), or abortion ( $34 \%$ vs $33 \%$ ) rates. This indicated that complete closure of the main branch of uterine arteries in surgical occlusion might present a greater risk of decreased perfusion of the uterus and placenta in pregnancy than more selective UAE. Therefore, it is currently not recommended to use this technique routinely outside clinical trials in patients who wish to become pregnant.

The main strength is the first study focusing on the effect of LM combined with LUAO on multiple myomas, and the thorough assessment of the intra- and postoperative outcomes. Before reaching conclusions in this study, several limitations should be discussed. First, this study is not prospective randomized, resulting in patient selection bias. There were differences in baseline characteristics between the two groups. Second, we treated patients according to their future fertility desire, which may expose the study to a few expected biases. This may have induced the difference in age of patients and the willingness of the operator to resect more fibroids when endometrial damage and uterine structure is not such a substantial concern. Third, the operation was performed by a single operator. Therefore, the data may not be applicable to all operators. In view of the aforementioned limitations, the results of our study should be interpreted with caution.

In conclusion, LM combined with LUAO was an effective and safe treatment for patients with multiple intramural symptomatic myomas who wished to retain their uteruses. The procedure's main benefits included less intraoperative bleeding and more thorough removal of myomas, which may be an important factor contributing to lower recurrence risks compared with LM alone. Based on the advantages of LUAO in myomectomy for patients with multiple myomas, further large multicenter prospective randomized controlled studies should be conducted.

\section{Ethics Approval and Consent to Participate}

This study was conducted with approval from the Ethics Committee of Beijing Obstetrics and Gynecology Hospital Capital Medical University. This study was conducted in accordance with the declaration of Helsinki. Written informed consent was obtained from all participants.

\section{Acknowledgments}

We would like to acknowledge the hard and dedicated work of all the staff that implemented the intervention and evaluation components of the study.

\section{Funding}

There is no funding to report.

\section{Disclosure}

The authors declare that they have no competing interests.

\section{References}

1. Yang W, Luo N, Ma L, Dai H, Cheng Z. The changes of surgical treatment for symptomatic uterine myomas in the past 15 years. J Minim Invasive Gynecol. 2018;7(1):10-15. doi:10.4103/GMIT. GMIT_11_17

2. Donnez J, Dolmans MM. Uterine fibroid management: from the present to the future. Hum Reprod Update. 2016;22(6):665-686. doi:10.1093/humupd/dmw023

3. Tranoulis A, Georgiou D, Alazzam M, Borley J. Combined laparoscopic uterine artery occlusion and myomectomy versus laparoscopic myomectomy: a direct-comparison meta-analysis of short- and long-term outcomes in women with symptomatic leiomyomas. J Minim Invasive Gynecol. 2019;26(5):826-837. doi:10.1016/j.jmig.2019.02.004

4. Dubuisson J, Ramyead L, Streuli I. The role of preventive uterine artery occlusion during laparoscopic myomectomy: a review of the literature. Arch Gynecol Obstet. 2015;291(4):737-743. doi:10.1007/ s00404-014-3546-4

5. Marret H, Fritel X, Ouldamer L, et al. Therapeutic management of uterine fibroid tumors: updated French guidelines. Eur J Obstet Gynecol Reprod Biol. 2012;165(2):156-164. doi:10.1016/j.ejogrb.2012.07.030

6. Vilos GA, Allaire C, Laberge PY, Leyland N; Special Contributors. The management of uterine leiomyomas. J Obstet Gynaecol Can. 2015;37(2):157-178. doi:10.1016/S1701-2163(15)30338-8

7. Munro MG, Critchley HO, Broder MS, Fraser IS. FIGO classification system (PALM-COEIN) for causes of abnormal uterine bleeding in nongravid women of reproductive age. Int $J$ Gynaecol Obstet. 2011;113(1):3-13. doi:10.1016/j.ijgo.2010.11.011

8. Laberge PY, Murji A, Vilos GA, et al. 389-Medical management of symptomatic uterine leiomyomas - an addendum. J Obstet Gynaecol Can. 2019;41(10):1521-1524. doi:10.1016/j.jogc.2019.01.010

9. Radosa MP, Owsianowski Z, Mothes A, et al. Long-term risk of fibroid recurrence after laparoscopic myomectomy. Eur J Obstet Gynecol Reprod Biol. 2014;180:35-39. doi:10.1016/j.ejogrb.2014.05.029

10. Ciavattini A, Clemente N, Delli Carpini G, Saccardi C, Borgato S, Litta P. Laparoscopic uterine artery bipolar coagulation plus myomectomy vs traditional laparoscopic myomectomy for "large" uterine fibroids: comparison of clinical efficacy. Arch Gynecol Obstet. 2017;296(6):1167-1173. doi:10.1007/s00404-017-4545-z 
11. Yang W, Cheng Z, Yu J, et al. Multicentre study to evaluate the clinical effects of laparoscopic uterine artery occlusion in combination with myomectomy to treat symptomatic uterine leiomyomas. Eur J Obstet Gynecol Reprod Biol. 2016;204:9-15. doi:10.1016/j. ejogrb.2016.05.033

12. Kwon YS, Roh HJ, Ahn JW, Lee SH, Im KS. Transient occlusion of uterine arteries in laparoscopic uterine surgery. JSLS. 2015;19(1): e2014.00189. doi:10.4293/JSLS.2014.00189

13. Yang W, Liu M, Liu L, et al. Uterine-sparing laparoscopic pelvic plexus ablation, uterine artery occlusion, and partial adenomyomectomy for adenomyosis. J Minim Invasive Gynecol. 2017;24 (6):940-945. doi:10.1016/j.jmig.2017.04.027

14. Li Y, Qi H, Ma W, et al. Temporarily blocking the uterine artery to dig out a diffused adenomyosis lesion treated laparoscopically. J Minim Invasive Gynecol. 2017;24(3):349-352. doi:10.1016/j. jmig.2016.09.014

15. Liu WM, Wang PH, Chou CS, Tang WL, Wang IT, Tzeng CR. Efficacy of combined laparoscopic uterine artery occlusion and myomectomy via minilaparotomy in the treatment of recurrent uterine myomas. Fertil Steril. 2007;87(2):356-361. doi:10.1016/j. fertnstert.2006.07.1497

16. Bae JH, Chong GO, Seong WJ, Hong DG, Lee YS. Benefit of uterine artery ligation in laparoscopic myomectomy. Fertil Steril. 2011;95(2):775-778. doi:10.1016/j.fertnstert.2010. 07.1079
17. Chang WC, Huang PS, Wang PH, et al. Comparison of laparoscopic myomectomy using in situ morcellation with and without uterine artery ligation for treatment of symptomatic myomas. JMinim Invasive Gynecol. 2012;19(6):715-721. doi:10.1016/j.jmig.2012.07.008

18. Jin L, Ji L, Shao M, Hu M. Laparoscopic myomectomy with temporary bilateral uterine artery and utero-ovarian vessels occlusion compared with traditional surgery for uterine fibroids: blood loss and recurrence. Gynecol Obstet Invest. 2019;84(6):548-554. doi:10.1159/000499494

19. Lee WL, Liu WM, Fuh JL, Tsai YC, Shih CC, Wang PH. Basal FSH level changes after different types of uterine vessel occlusion in the management of uterine fibroids. Fertil Steril. 2010;94(6):2286-2290. doi:10.1016/j.fertnstert.2009.11.038

20. Wang Y. Laparoscopic Transient Uterine Artery Occlusion and Myomectomy for Symptomatic Uterine Myoma as an Alternative to Hysterectomy. IntechOpen; 2015.

21. Alborzi S, Ghannadan E, Alborzi S, Alborzi M. A comparison of combined laparoscopic uterine artery ligation and myomectomy versus laparoscopic myomectomy in treatment of symptomatic myoma. Fertil Steril. 2009;92(2):742-747. doi:10.1016/j.fertnstert.2008.06.011

22. Michal M, Kubinova K, Maskova J, Horak P, Belsan T, Kuzel D. Uterine artery embolization versus laparoscopic uterine artery occlusion: the outcomes of a prospective, nonrandomized clinical trial. Cardiovasc Intervent Radiol. 2012;35(5):1041-1052. doi:10.1007/ s00270-012-0388-y
International Journal of General Medicine

\section{Publish your work in this journal}

The International Journal of General Medicine is an international, peer-reviewed open-access journal that focuses on general and internal medicine, pathogenesis, epidemiology, diagnosis, monitoring and treatment protocols. The journal is characterized by the rapid reporting of reviews, original research and clinical studies

\section{Dovepress}

across all disease areas. The manuscript management system is completely online and includes a very quick and fair peer-review system, which is all easy to use. Visit http://www.dovepress.com/ testimonials.php to read real quotes from published authors. 